\title{
Lifting Non-Finite Axiomatizability Results to Extensions of Process Algebras
}

\author{
Luca Aceto $^{1}$, Wan Fokkink ${ }^{2}$, Anna Ingolfsdottir ${ }^{1}$, and MohammadReza Mousavi ${ }^{3}$ \\ 1 Reykjavík University, Kringlan 1, IS-103, Reykjavík, Iceland \\ 2 Vrije Universiteit Amsterdam, NL-1081HV, The Netherlands \\ 3 Eindhoven University of Technology, NL-5600MB Eindhoven, The Netherlands
}

\begin{abstract}
This paper presents a general technique for obtaining new results pertaining to the non-finite axiomatizability of behavioral semantics over process algebras from old ones. The proposed technique is based on a variation on the classic idea of reduction mappings. In this setting, such reductions are translations between languages that preserve sound (in)equations and (in)equational proofs over the source language, and reflect families of (in)equations responsible for the non-finite axiomatizability of the target language. The proposed technique is applied to obtain a number of new non-finite axiomatizability theorems in process algebra via reduction to Moller's celebrated non-finite axiomatizability result for CCS. The limitations of the reduction technique are also studied.

This paper presents a general technique for obtaining new results pertaining to the non-finite axiomatizability of behavioral semantics over process algebras from old ones. The proposed technique is based on a variation on the classic idea of reduction mappings. In this setting, such reductions are translations between languages that preserve sound (in)equations and (in)equational proofs over the source language, and reflect families of (in)equations responsible for the non-finite axiomatizability of the target language. The proposed technique is applied to obtain a number of new non-finite axiomatizability theorems in process algebra via reduction to Moller's celebrated non-finite axiomatizability result for CCS. The limitations of the reduction technique are also studied.
\end{abstract}

\section{Introduction}

A classic and fundamental theoretical question in the study of algebras of processes is whether they afford a finite (in)equational axiomatization. Apart from being of foundational importance, (finite) axiomatizations of process semantics may form the basis for implementation verification using tools based on theorem-proving technology [10]. The first negative results concerning finite axiomatizability of process algebras go back to the Ph.D. thesis of Faron Moller [20], who showed that strong bisimilarity is not finitely based over CCS and over ACP without the left-merge operator. Since then, several other non-finite axiomatizability results have been obtained for a wide collection of very basic process algebras—see, e.g., [4] for a survey of such results.

In general, results concerning (non-)finite axiomatizability are very vulnerable to small changes in, and extensions of, the formalism under study. The addition of a single operator to a non-finitely axiomatizable formalism may make it finitely axiomatizable (e.g., adding the left-merge operator to the synchronization-free subset

Please use the following format when citing this chapter:

Aceto, L., et al., 2008, in IFIP International Federation for Information Processing, Volume 273; Fifth IFIP International Conference on Theoretical Computer Science; Giorgio Ausiello, Juhani Karhumäki, Giancarlo Mauri, Luke Ong; (Boston: Springer), pp. $301-316$. 
of CCS [9]). Conversely, the addition of a single operator may ruin the finite axiomatizability of a calculus (e.g., adding parallel composition to the sequential subset of CCS $[19,21])$. Also, apparently simple changes to the semantics of process calculi, e.g., adding aspects such as timing, may ruin the (non-)finite axiomatizability results and make their proofs obsolete (e.g., adding timing to synchronization-free CCS with left merge makes it non-finitely axiomatizable, as shown in [8]). Furthermore, proofs of non-finite axiomatizability results in the concurrency-theory literature are extremely delicate and error-prone; they are often rather long, and involve intricate syntactic arguments. Hence, we believe that it would be useful to find some general theorems that can be used to prove non-finite axiomatizability results. Such a general theory would allow one to relate non-finite axiomatizability theorems for different formalisms, and spare researchers (some of) the delicate technical analysis needed to adapt the proofs of such results. Despite some initial proposals, like the one in [2], it is fair to say that such a general theory is missing to date.

In this paper, we present a meta-theorem offering a general technique that can be used to prove non-finite axiomatizability results, and present some of its applications within concurrency theory. In this meta-theorem, we give sufficient criteria to obtain new non-finite axiomatizability results from known ones. The proposed technique is based on a variation on the classic idea of reduction mappings, which underlies the proofs of many classic undecidability results in computability theory and of lower bounds in complexity theory—see, e.g., [26] for a textbook presentation. In this setting, reductions are translations between languages that preserve sound (in)equations and (in)equational proofs over the source language, and reflect families of (in)equations responsible for the non-finite axiomatizability of the target language. We show the applicability of our reduction-based technique by obtaining several, to our knowledge novel, non-finite axiomatizability results for timed and stochastic process algebras. All these results are proved by showing that the existence of a finite axiomatization for the seven calculi we consider in this extended abstract would contradict a result of Moller's that entails the non-finite axiomatizability of strong bisimilarity over CCS. We also investigate some of the limitations of our reduction-based technique. In particular we exhibit a classic variation on CCS that is not finitely based, but whose non-finite axiomatizability cannot be shown by reduction to CCS modulo bisimilarity.

The paper is organized as follows. In Section 2, we review some preliminary definitions from universal algebra. Section 3 presents our reduction-based technique for proving non-finite axiomatizability results. In Section 4 we apply our approach to obtain seven new non-finite axiomatizability results. In Section 5, we illustrate the limitations of our proof methodology by presenting a non-finite axiomatizability result that cannot be proved using the strategy we employed to obtain the results in Section 4. These limitations can provide sources of inspiration for future improvements on our techniques. Finally, Section 6 concludes the paper and presents some directions for future and ongoing research.

Due to space restrictions, we have omitted most of the proofs of our results in this extended abstract. The reader is referred to [5] for full details and an in-depth coverage of the issues discussed in this paper. 


\section{Preliminaries}

We begin by recalling some basic notions from universal algebra that will be used throughout the paper. We refer the interested reader to, e.g., [14] for more information.

A signature $\Sigma$ is a set of function symbols $f, g, \ldots$ with fixed arities. A function symbol of arity zero is often called a constant (symbol). Given a signature $\Sigma$ and a set of variables $V$, terms $t, u, \ldots \in \mathscr{T}(\Sigma)$ are constructed inductively (from function symbols and variables) while respecting the arities of the function symbols. (In what follows, whenever we write a term $f\left(t_{1}, \ldots, t_{n}\right)$ we tacitly assume that the arity of $f$ is n.) Closed terms $p, q, \ldots \in \mathscr{C}(\Sigma)$ are terms that do not contain variables. We write $\equiv$ for syntactic equality over terms.

A precongruence $\precsim$ over $\mathscr{C}(\Sigma)$ is a substitutive preorder over $\mathscr{C}(\Sigma)$-that is, a preorder over $\mathscr{C}(\Sigma)$ that is preserved by all the function symbols in $\Sigma$. A congruence $\sim$ over $\mathscr{C}(\Sigma)$ is a substitutive equivalence relation. Each precongruence $\precsim$ over $\mathscr{C}(\Sigma)$ induces a congruence $\sim$ thus: $p \sim q$ iff $p \precsim q \precsim p$.

A (closed) substitution maps variables in $V$ to (closed) terms. For every term $t$ and substitution $\sigma$, the term $\sigma(t)$ is obtained by replacing every occurrence of a variable $x$ in $t$ by $\sigma(x)$. Note that $\sigma(t)$ is closed if $\sigma$ is a closed substitution. We write $\left[t_{1} / x_{1}, \ldots, t_{n} / x_{n}\right]$, where the $x_{i}(1 \leq i \leq n)$ are distinct variables, for the substitution mapping each variable $x_{i}$ to $t_{i}$, and acting like the identity function on all the other variables.

Given a relation $R$ over closed terms, for open terms $t$ and $u$, we define $t R u$ if $\sigma(t) R \sigma(u)$ for each closed substitution $\sigma$.

Consider a signature $\Sigma$. A set $E$ of equations $t=t^{\prime}$, where $t, t^{\prime} \in \mathscr{T}(\Sigma)$, is called an axiomatization (on $\mathscr{T}(\Sigma)$ ). We write $E \vdash t=t^{\prime}$ when $t=t^{\prime}$ is derivable from $E$ by the following set of inference rules.

$$
\begin{gathered}
(\text { refl }) \frac{E}{E \vdash t=t} \quad(\text { trans }) \frac{E \vdash t_{0}=t_{1} \quad E \vdash t_{1}=t_{2}}{E \vdash t_{0}=t_{2}} \\
(\text { cong }) \frac{E \vdash t_{1}=t_{1}^{\prime} \quad \ldots \quad E \vdash t_{n}=t_{n}^{\prime}}{E \vdash f\left(t_{1}, \ldots, t_{n}\right)=f\left(t_{1}^{\prime}, \ldots, t_{n}^{\prime}\right)} \quad(\mathbf{E}) \frac{}{E \vdash \sigma(t)=\sigma\left(t^{\prime}\right)} t=t^{\prime} \in E
\end{gathered}
$$

(Deduction rule (cong) is a rule schema with one instance for each function symbol $f$ in the signature $\Sigma$.) For axiomatizations $E$ and $E^{\prime}$, we write $E^{\prime} \vdash E$ when $E^{\prime} \vdash t=u$ for each $t=u \in E$. Above, we intentionally did not include the inference rule for symmetry, i.e., $(\mathbf{s y m m}) \frac{E \vdash t=t^{\prime}}{E \vdash t^{\prime}=t}$. Excluding (symm) does not restrict the applicability of our results by any measure. Any set of equations can be closed under symmetry by simply adding to it a symmetric copy of each equation, and this transformation preserves finiteness. (In what follows, we shall tacitly assume that each equational axiomatization is closed with respect to symmetry.) Furthermore, the omission of the rule for symmetry allows us to deal with axiomatizations for precongruences, which are not necessarily symmetric relations. When working with precongruences, our axiomatizations consist of inequations $t \leq t^{\prime}$ between terms. 
Given a congruence $\sim \subseteq \mathscr{T}(\Sigma) \times \mathscr{T}(\Sigma)$, an equation $t=t^{\prime}$ is sound modulo $\sim$ when $t \sim t^{\prime}$. An axiomatization is sound modulo $\sim$ if each of its equations is sound modulo $\sim$. An axiomatization $E$ is complete modulo $\sim$ if for each sound equation $t=t^{\prime}$, it holds that $E \vdash t=t^{\prime} . E$ is ground-complete modulo $\sim$ if for each closed sound equation $p=q$, it holds that $E \vdash p=q$. We say that $\sim$ is finitely based over $\mathscr{T}(\Sigma)$ if there is a finite, sound and complete axiomatization for $\mathscr{T}(\Sigma)$ modulo $\sim$. Similar definitions apply to precongruences and inequational axiomatizations.

\section{The Reduction Theorem}

Our aim in this section will be to present a general result that will allow us to lift nonfinite axiomatizability results from one process algebra to another. Throughout this section, we fix two signatures $\Sigma_{o}$ and $\Sigma_{e}$, a common set of variables $V$ and two precongruences $\precsim_{o}$ and $\precsim_{e}$ over $\mathscr{T}\left(\Sigma_{o}\right)$ and $\mathscr{T}\left(\Sigma_{e}\right)$, respectively. Intuitively, the signature $\Sigma_{o}$ stands for the collection of operations in an original process language for which we already have a non-finite axiomatizability result modulo the precongruence $\precsim o$. On the other hand, the signature $\Sigma_{e}$ stands for the collection of operations in an extended process language for which we intend to prove a non-finite axiomatizability result modulo the precongruence $\precsim e$. Since a congruence is a symmetric precongruence, all the results we present in the remainder of this section apply equally well when any of $\precsim$ o and $\precsim e$ is a congruence relation.

Consider a mapping $\widehat{-}: \mathscr{T}\left(\Sigma_{e}\right) \rightarrow \mathscr{T}\left(\Sigma_{o}\right)$. For an axiomatization $E$ over $\mathscr{T}\left(\Sigma_{e}\right)$, we define the axiomatization $\widehat{E}$ over $\mathscr{T}\left(\Sigma_{o}\right)$ to be $\{\widehat{t} \leq \widehat{u} \mid t \leq u \in E\}$.

Definition 1. A function $\widehat{\widehat{-}}: \mathscr{T}\left(\Sigma_{e}\right) \rightarrow \mathscr{T}\left(\Sigma_{o}\right)$ is a reduction from $\mathscr{T}\left(\Sigma_{e}\right)$ to $\mathscr{T}\left(\Sigma_{o}\right)$, when for all $t, u \in \mathscr{T}\left(\Sigma_{e}\right)$,

1. $t \precsim e u \Rightarrow \widehat{t} \precsim o \widehat{u}$ (that is, - preserves sound inequations), and

2. $E \vdash t \leq u \Rightarrow \widehat{E} \vdash \widehat{t} \leq \widehat{u}$, for each axiomatization $E$ on $\mathscr{T}\left(\Sigma_{e}\right)$ (that is, $\widehat{-}$ preserves provability).

Definition 2. Let $E$ be an axiomatization over $\mathscr{T}\left(\Sigma_{o}\right)$. A reduction $\widehat{-}$ is $E$-reflecting, when for each $t \leq u \in E$, there exists an inequation $t^{\prime} \leq u^{\prime}$ over $\mathscr{T}\left(\Sigma_{e}\right)$ that is sound modulo $\precsim e$ such that $\widehat{t^{\prime}} \equiv t$ and $\widehat{u^{\prime}} \equiv u$. The reduction $\widehat{-}$ is called ground E-reflecting if for each closed inequation $p \leq q \in E$, there exists a closed inequation $p^{\prime} \leq q^{\prime}$ on $\mathscr{T}\left(\Sigma_{e}\right)$ that is sound modulo $\precsim e$ such that $\widehat{p^{\prime}} \equiv p$ and $\widehat{q^{\prime}} \equiv q$.

We are now ready to state the general tool that we shall use in this paper to lift nonfinite axiomatizability results from $\mathscr{T}\left(\Sigma_{o}\right)$ modulo $\precsim o$ to $\mathscr{T}\left(\Sigma_{e}\right)$ modulo $\precsim e$.

Theorem 1. Assume that there is a set of inequations $E$ on $\mathscr{T}\left(\Sigma_{o}\right)$ that is sound modulo $\precsim$ ond that is not provable from any finite sound axiomatization on $\mathscr{T}\left(\Sigma_{o}\right)$. If there exists an E-reflecting reduction from $\mathscr{T}\left(\Sigma_{e}\right)$ to $\mathscr{T}\left(\Sigma_{o}\right)$, then $\precsim e$ is not finitely based over $\mathscr{T}\left(\Sigma_{e}\right)$. 
The above theorem gives us a general technique to lift non-finite axiomatizability results from a language $\mathscr{T}\left(\Sigma_{o}\right)$ modulo $\precsim_{o}$ to a language $\mathscr{T}\left(\Sigma_{e}\right)$ modulo $\precsim_{e}$. Indeed, suppose that we know that a precongruence $\precsim$ is not finitely based over $\mathscr{T}\left(\Sigma_{o}\right)$. Typically, such a negative result is shown by exhibiting an infinite collection $E$ of sound inequations that cannot be proved from any finite sound axiomatization over $\Sigma_{o}$. (See, e.g., $[1,3,4,6,8,11,12,20,22]$ and the references therein.) In the light of the above theorem, to show that $\precsim e$ is not finitely based over $\mathscr{T}\left(\Sigma_{e}\right)$ it suffices only to exhibit an $E$-reflecting reduction from $\mathscr{T}\left(\Sigma_{e}\right)$ to $\mathscr{T}\left(\Sigma_{o}\right)$.

As the examples we present in Section 4 will show, Theorem 1, albeit not technically complex, is widely applicable. In all our applications of Theorem 1, the reduction from $\Sigma_{e}$ to $\Sigma_{o}$ is defined inductively on the structure of terms. Since such "structural" reductions play an important role in the remainder of the paper, we now proceed to define them precisely and to state a very useful property such reductions afford.

Definition 3. A mapping $ح: \mathscr{T}\left(\Sigma_{e}\right) \rightarrow \mathscr{T}\left(\Sigma_{o}\right)$ is structural if

1. it is the identity on variables, i.e., $\widehat{x} \equiv x$ for each $x \in V$,

2. it does not introduce new variables, i.e., $\operatorname{vars}\left(f\left(\widehat{x_{1}, \ldots,} x_{n}\right)\right) \subseteq\left\{x_{1}, \ldots, x_{n}\right\}$, for each $f \in \Sigma_{e}$ and sequence of distinct $x_{1}, \ldots, x_{n} \in V$, and

3. it is defined compositionally, i.e., $f\left(\widehat{t_{1}, \ldots,} t_{n}\right) \equiv f\left(\widehat{x_{1}, \ldots,} x_{n}\right)\left[\widehat{t}_{1} / x_{1}, \ldots, \widehat{t_{n}} / x_{n}\right]$, for each $f \in \Sigma_{e}$, and sequences of distinct $x_{1}, \ldots, x_{n} \in V$ and of $t_{1}, \ldots, t_{n} \in \mathscr{T}\left(\Sigma_{e}\right)$.

Lemma 1. Let $\widehat{\mathcal{Z}}: \mathscr{T}\left(\Sigma_{e}\right) \rightarrow \mathscr{T}\left(\Sigma_{o}\right)$ be a structural mapping. Then $\widehat{\sigma(t)} \equiv \widehat{\sigma}(\widehat{t})$, for each term $t \in \mathscr{T}\left(\Sigma_{e}\right)$ and each substitution $\sigma$ over $\Sigma_{e}$.

The following theorem shows that, if the reduction is structural, one can dispense with proving item 2 of Definition 1. Since each reduction we consider in this paper is structural, this result eases our applications of Theorem 1 considerably.

Theorem 2. A structural mapping satisfies item 2 of Definition 1.

If the collection of equations $E$ mentioned in the statement of Theorem 1 is closed, then one can prove impossibility of a finite ground-complete axiomatization of $\precsim_{e}$ over $\mathscr{T}\left(\Sigma_{e}\right)$, which is a stronger result than Theorem 1.

Theorem 3. Assume that there is a set of closed equations $E$ that is sound modulo $\precsim$, and that is not provable from any finite axiomatization over $\mathscr{T}\left(\Sigma_{o}\right)$ that is sound modulo $\precsim$. If there exists a ground E-reflecting reduction from $\Sigma_{e}$ to $\Sigma_{o}$, then there exists no sound and ground-complete finite axiomatization for $\precsim_{e}$ over $\mathscr{T}\left(\Sigma_{e}\right)$.

For structural reductions whose source is a language over a signature that contains at least one constant, in order to apply Theorem 3 it suffices to show that the reduction is $E$-reflecting by the following theorem. Thus, if the collection of equations $E$ is closed and the reduction is structural, one can readily obtain impossibility of a finite groundcomplete axiomatization without any further work (by showing that the premises of Theorem 1 hold).

Theorem 4. An E-reflecting structural reduction $\widehat{-}$ is also ground E-reflecting, provided that the signature $\Sigma_{e}$ contains at least one constant symbol. 
The set of basic equations that we shall use throughout the rest of this paper in our applications of Theorem 1 is closed and, furthermore, all our reductions are structural; thus, all the impossibility results we present in the subsequent section hold for groundcomplete as well as complete axiomatizations.

\section{Applications}

In this section, we take a well-known non-finite axiomatizability result in the setting of process algebra due to Moller [20, 21], and use Theorem 1 to establish other, to the best of our knowledge novel, non-finite axiomatizability results for several notions of behavioral (pre)congruences over other process algebras. A brief comparison between the full proof of the original result in [20,21] and those based on Theorem 1 presented here (and in the full version of this paper [5]) reveals that our proofs are substantially more concise and simpler than direct proofs. This is despite the fact that the calculi and notions of (pre)congruence treated henceforth are more sophisticated than the ones treated in $[20,21]$.

Consider the subset of CCS [19] with the following syntax.

$$
P::=\mathbf{0}|a . P| P+P|P| \mid P
$$

Note that here a.P stands for one unary operator (action-prefixing with one particular action $a$ ) and not, as it is customary, for a collection of unary operators. Henceforth, we denote the signature of the above-mentioned calculus by $\Sigma_{o}$ since that fragment of CCS will be the target language in all the applications of Theorem 1 to follow.

The operational semantics of the calculus above is given by the following SOS rules.

$$
\text { (a) } \frac{x_{0} \stackrel{a}{a} y}{a . x \stackrel{a}{\rightarrow} x}(\mathbf{c 0}) \frac{x_{0} \stackrel{a}{\rightarrow} y_{0}}{x_{0}+x_{1} \stackrel{a}{\rightarrow} y}(\mathbf{p 0}) \frac{\stackrel{a}{\rightarrow} y_{0} \| x_{1}}{x_{0} \| x_{1}}
$$

Note that we have omitted the symmetric versions of $(\mathbf{c 0})$ and (p0), for brevity; furthermore, since there is only one action (and no co-action) in our signature, the standard SOS rule for communication in CCS can be safely omitted.

Definition 4. A symmetric relation $R \subseteq \mathscr{C}\left(\Sigma_{o}\right) \times \mathscr{C}\left(\Sigma_{o}\right)$ is a strong bisimulation when for all $(p, q) \in R$ and $p^{\prime} \in \mathscr{C}\left(\Sigma_{o}\right)$, if $p \stackrel{a}{\rightarrow} p^{\prime}$ then there exists a $q^{\prime}$ such that $q \stackrel{a}{\rightarrow} q^{\prime}$ and $\left(p^{\prime}, q^{\prime}\right) \in R$. Two closed terms $p$ and $q$ are strongly bisimilar (or just bisimilar), denoted by $p \biguplus_{b} q$, when there exists a strong bisimulation $R$ such that $(p, q) \in R$.

Moller showed in $[20,21]$ that strong bisimilarity affords no finite ground-complete axiomatization over the above calculus. His negative result was a corollary of the following stronger theorem.

Theorem 5 (Moller [20, 21]). There is no finite axiomatization over the signature $\Sigma_{o}$ that is sound modulo strong bisimilarity and proves all the equations in the set $\mathscr{M}$ defined below: 


$$
\left\{a^{1} \|\left(a^{1}+a^{2}+\cdots+a^{n}\right)=a \cdot\left(a^{1}+a^{2}+\cdots+a^{n}\right)+a^{2}+a^{3}+\cdots+a^{n+1} \mid n \geq 1\right\},
$$

where $a^{i}=\underbrace{a \ldots \ldots a}_{i \text { times }} \mathbf{0}$, for each $i \geq 1$.

In the remainder of this section, we use Theorems 1 and 5 to obtain other non-finite axiomatizability results, with the aforementioned fragment of CCS as the target language for our reductions.

\subsection{Discrete-time CCS and Timed Bisimilarity}

Timed CCS is a timed extension of CCS proposed by Wang Yi [27]. In [8], we proved some non-finite axiomatizability results for Timed CCS modulo timed bisimilarity under the assumption that the underlying time domain satisfy a density property, and left open whether those results carry over to the discrete-time fragment of Timed CCS (referred to as DiTCCS in what follows). In this section, we instantiate our reduction theorem to show that a finite sound and ground-complete axiomatization for DiTCCS modulo timed bisimilarity does not exist.

Let $A$ be a set of actions that contains the action $a$. Following Milner, we write $\bar{A}$ for the set of complementary actions $\{\bar{b} \mid b \in A\}$, and assume that $\overline{\bar{\alpha}}=\alpha$ for each $\alpha \in A \cup \bar{A}$. The internal action is denoted by $\tau \notin A \cup \bar{A}$. The syntax of DiTCCS is given by the grammar:

$$
P::=\mathbf{0}|\mu . P| \varepsilon(d) . P|P+P| P \| P,
$$

where $\mu . P$ is a set of unary operators, one for each $\mu \in A \cup \bar{A} \cup\{\tau\}$, and $\varepsilon(d) . P$ is a set of unary operators, one for each $d \in \mathbb{N}=\{1,2, \ldots\}$. In this subsection, we refer to the signature of DiTCCS as $\Sigma_{e}$ since we use this language as our source language in applying Theorem 1. The operational semantics of DiTCCS is given by the following set of SOS rules, where $\alpha \in A \cup \bar{A}, \mu \in A \cup \bar{A} \cup\{\tau\}$ and $d, e \in \mathbb{N}$.

$$
\begin{aligned}
& (\mathbf{t n}) \frac{}{\mathbf{0} \stackrel{\varepsilon(d)}{\longrightarrow} \mathbf{0}} \\
& \text { (a) } \frac{\mu}{\mu \cdot x \stackrel{\mu}{\rightarrow} x} \\
& (\mathbf{t d 0}) \frac{}{\varepsilon(d) \cdot x \stackrel{\varepsilon(d)}{\rightarrow} x} \\
& (\mathbf{t d 1}) \overline{\varepsilon(d+e) \cdot x \stackrel{\varepsilon(d)}{\rightarrow} \varepsilon(e) \cdot x} \\
& \text { (c0) } \frac{x_{0} \stackrel{\mu}{\rightarrow} y}{x_{0}+x_{1} \stackrel{\mu}{\rightarrow} y} \\
& \text { (tc) } \frac{x_{0} \stackrel{\varepsilon(d)}{\longrightarrow} y_{0} \quad x_{1} \stackrel{\varepsilon(d)}{\longrightarrow} y_{1}}{x_{0}+x_{1} \stackrel{\varepsilon(d)}{\longrightarrow} y_{0}+y_{1}} \\
& \text { (p2) } \frac{x_{0} \stackrel{\alpha}{\rightarrow} y_{0} \quad x_{1} \stackrel{\bar{\alpha}}{\rightarrow} y_{1}}{x_{0}\left\|x_{1} \stackrel{\tau}{\rightarrow} y_{0}\right\| y_{1}} \\
& (\mathbf{t p}) \frac{x_{0} \stackrel{\varepsilon(d)}{\longrightarrow} y_{0} \quad x_{1} \stackrel{\varepsilon(d)}{\longrightarrow} y_{1}}{x_{0}\left\|x_{1} \stackrel{\varepsilon(d)}{\longrightarrow} y_{0}\right\| y_{1}} \\
& \text { (ta) } \frac{\varepsilon(d)}{\alpha \cdot x \stackrel{(d)}{\longrightarrow} \alpha \cdot x} \\
& (\operatorname{td2}) \frac{x \stackrel{\varepsilon(e)}{\longrightarrow} y}{\varepsilon(d) \cdot x \stackrel{\varepsilon(d+e)}{\longrightarrow} y} \\
& (\mathbf{p 0}) \frac{x_{0} \stackrel{\mu}{\rightarrow} y_{0}}{x_{0}\left\|x_{1} \stackrel{\mu}{\rightarrow} y_{0}\right\| x_{1}} \\
& \operatorname{Sort}_{d}\left(x_{0}\right) \cap \overline{\operatorname{Sort}_{d}\left(x_{1}\right)}=\emptyset
\end{aligned}
$$

These rules define transitions between closed DiTCCS terms. (Again, we have omitted the symmetric versions of (c0) and (p0).) The side condition in rule (tp) uses the 
timed sort $\operatorname{Sort}_{d}(p)$, where $p$ is a closed DiTCCS term and $d \in \mathbb{N}$, which is defined thus: $\operatorname{Sort}_{d}(p)=\left\{\alpha \in A \cup \bar{A} \mid p \stackrel{\varepsilon(e)}{\longrightarrow} p^{\prime} \stackrel{\alpha}{\rightarrow}\right.$ for some $p^{\prime}$ and $\left.e<d\right\}$.

The notion of equivalence over DiTCCS we shall consider in what follows is timed bisimilarity, denoted by $\biguplus_{t}$. Timed bisimilarity is just bisimilarity over the labelled transition system whose states are terms in $\mathscr{C}\left(\Sigma_{e}\right)$ and whose transitions are of the form $p \stackrel{\chi}{\rightarrow} p^{\prime}$, where $\chi \in A \cup \bar{A} \cup\{\tau\} \cup\{\varepsilon(d) \mid d \in \mathbb{N}\}$. It is well known that $\biguplus_{t}$ is a congruence over DiTCCS; see, e.g., [27, Theorem 5.1], where the congruence result is stated for dense-time Timed CCS.

Theorem 6. DiTCCS affords no finite ground-complete axiomatization modulo $\biguplus_{t}$.

We prove the above result using Theorem 1. To this end, we begin by defining the following translation $\widehat{\widehat{-}}: \mathscr{T}\left(\Sigma_{e}\right) \rightarrow \mathscr{T}\left(\Sigma_{o}\right)$.

$$
\begin{array}{rcrl}
\widehat{\mathbf{0}}=\mathbf{0} & \widehat{x}=x & \widehat{\mu \cdot p} & = \begin{cases}a \cdot \widehat{p} & \text { if } \mu=a, \\
\mathbf{0} & \text { if } \mu \neq a .\end{cases} \\
\widehat{\varepsilon(d) \cdot p} & =\mathbf{0} & \widehat{p+q}=\widehat{p}+\widehat{q} & \widehat{p \| q}=\widehat{p} \| \widehat{q}
\end{array}
$$

\section{Lemma 2. The mapping $\widehat{-}$ defined above is structural.}

Consider now the set of Moller's equations $\mathscr{M}$, which are sound over CCS modulo bisimilarity. In order to prove that timed bisimilarity is not finitely based over DiTCCS, by Theorem 1 it suffices only to show the following statements:

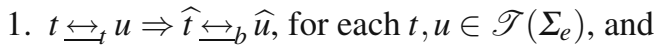

2. - is $\mathscr{M}$-reflecting.

Note that, for each axiomatization $E$ over the signature of DiTCCS,

$$
E \vdash t=u \Rightarrow \widehat{E} \vdash \widehat{t}=\widehat{u}
$$

holds by Theorem 2 since $\_$is structural (Lemma 2). Therefore, once we prove the two statements above, Theorem 6 indeed follows as a corollary of Theorem 1.

Next, we give the proofs of the above two statements.

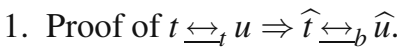

In order to prove this statement, it suffices to show that the relation

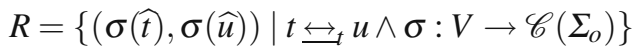

is a bisimulation. To this end, observe, first of all, that $R$ is symmetric. In order to prove that $R$ satisfies the transfer property in Definition 4, we shall make use of the following two claims, whose proof will be given later.

a. For all $p \in \mathscr{C}\left(\Sigma_{e}\right)$ and $p^{\prime} \in \mathscr{C}\left(\Sigma_{o}\right)$, if $\widehat{p} \stackrel{a}{\rightarrow} p^{\prime}$ with respect to the operational semantics of CCS, then there exists some $p^{\prime \prime} \in \mathscr{C}\left(\Sigma_{e}\right)$ such that $p \stackrel{a}{\rightarrow} p^{\prime \prime}$, with respect to the operational semantics of DiTCCS, and $\widehat{p^{\prime \prime}} \equiv p^{\prime}$. 
b. For all $p, p^{\prime} \in \mathscr{C}\left(\Sigma_{e}\right)$, if $p \stackrel{a}{\rightarrow} p^{\prime}$ with respect to the operational semantics of DiTCCS, then $\widehat{p} \stackrel{a}{\rightarrow} \widehat{p}^{\prime}$ with respect to the operational semantics of CCS.

Assume now that $\sigma(\widehat{t}) R \sigma(\widehat{u})$ and $\sigma(\widehat{t}) \stackrel{a}{\rightarrow} p_{0}^{\prime}$. By Lemmas 1 and $2, \sigma(\widehat{t}) \equiv \widehat{\sigma(t)}$. It follows from item 1a above that $\sigma(t) \stackrel{a}{\rightarrow} p_{0}$, for some $p_{0}$ such that $\widehat{p_{0}} \equiv p_{0}^{\prime}$. Furthermore, as $t$ and $u$ are timed bisimilar, $\sigma(u) \stackrel{a}{\rightarrow} p_{1}$, for some $p_{1}$ such that $p_{0} \biguplus_{t} p_{1}$. From item $1 \mathrm{~b}$ and Lemmas $1-2$, we have that $\sigma(\widehat{u}) \equiv \widehat{\sigma(u)} \stackrel{a}{\rightarrow} \widehat{p_{1}}$ and, by the definition of $R$, we may conclude that $p_{0}^{\prime}=\widehat{p_{0}} R \widehat{p_{1}}$, which was to be shown.

In order to complete the proof of this statement, we are therefore left to show items $1 \mathrm{a}$ and $1 \mathrm{~b}$. This we now proceed to do.

a. Proof of item 1a.

We prove this claim by an induction on the structure of $p$, and only detail the argument for two representative cases.

- Assume that $p \equiv \mu . p_{0}$. Then $p$ must be of the form $a . p_{0}$ (in order for $\widehat{p}$ to make an $a$-transition) and thus, $\widehat{p}=a \cdot \widehat{p_{0}} \stackrel{a}{\rightarrow} \widehat{p_{0}}=p^{\prime}$. The claim then follows since $a \cdot p_{0} \stackrel{a}{\rightarrow} p_{0}$.

- Assume that $p \equiv p_{0}+p_{1}$. Then $\widehat{p} \equiv \widehat{p_{0}}+\widehat{p_{1}}$. Suppose, without loss of generality, that the transition $\widehat{p_{0}}+\widehat{p_{1}} \stackrel{a}{\rightarrow} p^{\prime}$ is due to an application of rule $(\mathbf{c 0})$; thus, $\widehat{p_{0}} \stackrel{a}{\rightarrow} p^{\prime}$. It then follows from the induction hypothesis that $p_{0} \stackrel{a}{\rightarrow} p^{\prime \prime}$ for some $p^{\prime \prime}$ such that $\widehat{p^{\prime \prime}} \equiv p^{\prime}$. By applying deduction rule (c0), we obtain $p \equiv p_{0}+p_{1} \stackrel{a}{\rightarrow} p^{\prime \prime}$.

b. Proof of item $1 b$.

By an induction on the depth of the proof for $p \stackrel{a}{\rightarrow} p^{\prime}$. We distinguish the following cases based on the last deduction rule applied to obtain $p \stackrel{a}{\rightarrow} p^{\prime}$.

(a) In this case, $p$ is of the form $a \cdot p_{0}$ and $p^{\prime} \equiv p_{0}$ Thus, using to the same deduction rule in the semantics of CCS, we have $\widehat{p} \equiv a \cdot \widehat{p_{0}} \stackrel{a}{\rightarrow} \widehat{p_{0}}$.

(c0) Then $p \equiv p_{0}+p_{1}$ and $p_{0} \stackrel{a}{\rightarrow} p^{\prime}$ by a shorter inference. It follows from the induction hypothesis that $\widehat{p_{0}} \stackrel{a}{\rightarrow} \widehat{p^{\prime}}$ and, using rule (c0) in the semantics of CCS, we infer that $\widehat{p_{0}}+\widehat{p_{1}} \stackrel{a}{\rightarrow} \widehat{p^{\prime}}$. Furthermore, by the definition of $\widehat{\text {-, }}$, we have that $\widehat{p} \equiv \widehat{p_{0}}+\widehat{p}_{1}$.

The cases for deduction rules $(\mathbf{c 1}),(\mathbf{p} \mathbf{0})$ and $(\mathbf{p} \mathbf{1})$ are similar to the case of $(\mathbf{c 0})$.

The proof of the first statement is now complete.

2. Proof of the fact that $\_$is $\mathscr{M}$-reflecting.

We show that all axioms in $\mathscr{M}$ are sound modulo $\biguplus_{t}$. Since $\widehat{-}$ is the identity over CCS terms, the statement then follows immediately. To this end, we prove the following two claims.

a. For each $p \in \mathscr{C}\left(\Sigma_{o}\right)$ and positive integer $d, p \stackrel{\varepsilon(d)}{\rightarrow} p^{\prime}$ iff $p \equiv p^{\prime}$. We prove this claim by an induction on the structure of $p$. The cases for $\mathbf{0}$ and $a . p_{0}$ follow from deduction rules $(\mathbf{t n})$ and $(\mathbf{t a})$, respectively. The cases for $p_{0}+p_{1}$ and $p_{0} \| p_{1}$ follow from the induction hypothesis, and (tc) and (tp), respectively.

b. For each $p, q \in \mathscr{C}\left(\Sigma_{o}\right)$, if $p \biguplus_{b} q$ then $p \biguplus_{t} q$. 
We show that $\unlhd_{b}$ is a timed bisimulation. To this end, note, first of all, that the relation $\unlhd_{b}$ is symmetric. Assume now that $p \stackrel{a}{\rightarrow} p^{\prime}$ and $p \unlhd_{b} q$. Since $\unlhd_{b}$ is a bisimulation, it follows that $q \stackrel{a}{\rightarrow} q^{\prime}$ (with respect to the semantics of CCS, and

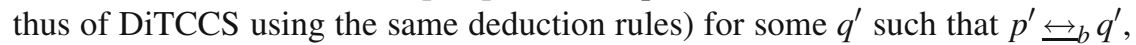
and we are done. That delay transitions of $p$ may be matched by $q$ follows trivially from the previous item.

Since all the provisos of Theorem 1 are met, Theorem 6 follows.

\subsection{Temporal CCS}

In the paper [23], Moller and Tofts proposed another timed extension of Milner's CCS, which they called Temporal Calculus of Communicating Systems (referred to as TCCS $\mathrm{MT}_{\mathrm{T}}$ in what follows to avoid any confusion with Wang Yi's Timed CCS), and studied its semantics theory modulo timed bisimilarity. Our order of business in this section is to use our reduction-based method to show that timed bisimilarity affords no finite ground-complete axiomatization over TCCS $_{\mathrm{MT}}$.

For our purposes in this section, $\mathrm{TCCS}_{\mathrm{MT}}$ is the language generated by the following grammar:

$$
P::=\mathbf{0}|\mu . P|(d) . P|\delta . P| P+P|P \oplus P| P \| P,
$$

where $\mu . P$ is a set of unary operators, one for each $\mu \in A \cup \bar{A} \cup\{\tau\}$, and $(d)$.P is a set of unary operators, one for each positive integer $d$. The intuition underlying each of the operators in the signature of TCCS $\mathrm{MT}_{\mathrm{MT}}$ is carefully described in [23, Pages 402-403]. For the sake of clarity, however, we find it useful to mention that:

- process terms of the form $\mathbf{0}$ or $\alpha$.p cannot delay, unlike in DiTCCS;

- $(d) \cdot p$ behaves exactly like $\varepsilon(d) \cdot p$ in DiTCCS;

$-\delta . p$ describes a process which behaves like $p$, but is willing to wait any amount to time before doing so; and

- $p \oplus q$ is a "weak choice" between $p$ and $q$. The choice between $p$ and $q$ is made upon performance of an action from either of the two processes, or at the occurrence of a time delay which can only be performed by one of the processes. By way of example, as $a . p$ cannot delay, a process of the form $a . p \oplus(1) .0$ will be transformed into $\mathbf{0}$ after a delay of one time unit.

In order to define the operational semantics of the weak choice operator, the Plotkinstyle rules for that operator from [23] make use of the function maxdelay(), which associates a non-negative integer or $\omega$ with each closed TCCS $_{\mathrm{MT}}$ term. The function maxdelay() is defined by structural induction on terms as follows:

$$
\begin{aligned}
& \operatorname{maxdelay}(\mathbf{0})=\operatorname{maxdelay}(\mu \cdot p)=0 \quad \operatorname{maxdelay}(\delta \cdot p)=\omega \\
& \operatorname{maxdelay}(p+q)=\operatorname{maxdelay}(p \| q)=\min (\operatorname{maxdelay}(p), \operatorname{maxdelay}(q)) \\
& \operatorname{maxdelay}(p \oplus q)=\max (\operatorname{maxdelay}(p), \operatorname{maxdelay}(q)) .
\end{aligned}
$$




$$
\begin{aligned}
& \overline{\delta . x \stackrel{\varepsilon(d)}{\longrightarrow} \delta . x} \\
& \frac{x \stackrel{\varepsilon(e)}{\rightarrow} y}{(d) \cdot x \stackrel{\varepsilon(d+e)}{\rightarrow} y}
\end{aligned}
$$

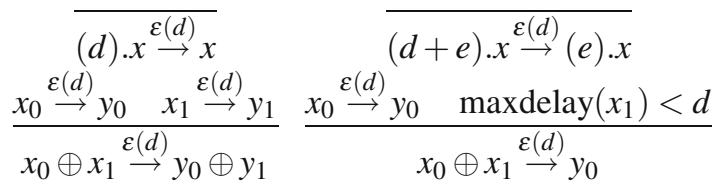

$$
\begin{aligned}
& \frac{x_{1} \stackrel{\varepsilon(d)}{\rightarrow} y_{1} \quad \text { maxdelay }\left(x_{0}\right)<d}{x_{0} \oplus x_{1} \stackrel{\varepsilon(d)}{\longrightarrow} y_{1}} \\
& \frac{x_{0} \stackrel{\varepsilon(d)}{\rightarrow} y_{0} \quad x_{1} \stackrel{\varepsilon(d)}{\longrightarrow} y_{1}}{x_{0}+x_{1} \stackrel{\varepsilon(d)}{\longrightarrow} y_{0}+y_{1}} \\
& \frac{x_{0} \stackrel{\varepsilon(d)}{\longrightarrow} y_{0} \quad x_{1} \stackrel{\varepsilon(d)}{\longrightarrow} y_{1}}{x_{0}\left\|x_{1} \stackrel{\varepsilon(d)}{\longrightarrow} y_{0}\right\| y_{1}}
\end{aligned}
$$

Table 1 Rules defining the delay transitions $\stackrel{\varepsilon(d)}{\rightarrow}$ over $\operatorname{TCCS}_{\mathrm{MT}}(d \in \mathbb{N})$

The operational semantics of closed TCCS $\mathrm{MT}_{\mathrm{MT}}$ terms is given by means of two types of transitions, namely actions transitions $\stackrel{\mu}{\rightarrow}$ with $\mu \in A \cup \bar{A} \cup\{\tau\}$ and delay transitions $\stackrel{\varepsilon(d)}{\rightarrow}$, with $d \in \mathbb{N}$. The transition relations $\stackrel{\mu}{\longrightarrow}$ are defined as for DiTCCS, with the proviso that

$-(d) \cdot p$ has no outgoing action transitions,

- $p \oplus q$ has the same outgoing action transitions as $p+q$, and

- the action transitions of $\delta . p$ are exactly those of $p-$ i.e., they are those provable using the rules

$$
\frac{x \stackrel{\mu}{\rightarrow} y}{\delta . x \stackrel{\mu}{\rightarrow} y} \quad(\mu \in A \cup \bar{A} \cup\{\tau\}) .
$$

On the other hand, the transition relations $\stackrel{\varepsilon(d)}{\longrightarrow}$ are the least relations satisfying the rules on Table 1 . Closed TCCS $\mathrm{MT}_{\mathrm{T}}$ terms are considered modulo timed bisimilarity $\biguplus_{t}$ (as defined in Section 4.1). Timed bisimilarity is a congruence over TCCS $\mathrm{MT}_{\mathrm{MT}}$ as shown in [23, Proposition 3.4].

\section{Theorem 7. TCCS $S_{M T}$ affords no finite ground-complete axiomatization modulo $\biguplus_{t}$.}

In the remainder of this subsection, we prove the above result using Theorem 1. To this end, we begin by defining the following translation $\widehat{\text { _ from open }} \mathrm{TCCS}_{\mathrm{MT}}$ terms to open CCS terms.

$$
\begin{aligned}
& \widehat{\mathbf{0}}=\mathbf{0} \quad \widehat{x}=x \quad \widehat{\delta \cdot p}=\widehat{p} \\
& \widehat{a \cdot p}=a \cdot \widehat{p} \quad \widehat{\mu \cdot p}=\mathbf{0} \text { for } \mu \neq a \quad \widehat{(d) \cdot p}=\mathbf{0} \\
& \widehat{p+q}=\widehat{p}+\widehat{q} \quad \widehat{p \oplus q}=\widehat{p}+\widehat{q} \quad \widehat{p \| q}=\widehat{p} \| \widehat{q}
\end{aligned}
$$

Remark 1. Note that the mapping obtained from the one defined above by associating $\mathbf{0}$ to $\widehat{p \oplus q}$ would not be a reduction, since it does not preserve valid equations. For example, the valid equation $x \oplus x=x$ would not be preserved by such a mapping.

Lemma 3. The mapping $\widehat{-}$ defined above is structural. 
Consider now the set of Moller's equations $\mathscr{M}$, which are sound over CCS modulo bisimilarity. In order to prove that timed bisimilarity is not finitely based over TCCS $_{\mathrm{MT}}$, by Theorem 1 it suffices only to show the following statements:

1. $t \biguplus_{t} u$ implies $\widehat{t} \biguplus_{b} \widehat{u}$, for all TCCS $\mathrm{MT}_{\mathrm{M}}$ terms $t, u$, and

2. $\widehat{\text { is }} \mathscr{M}$-reflecting.

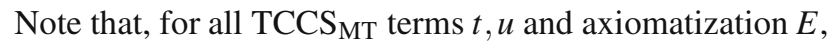

$$
E \vdash t=u \Rightarrow \widehat{E} \vdash \widehat{t}=\widehat{u}
$$

holds by Theorem 2 since $\widehat{-}$ is structural (Lemma 3 ). Therefore, once we prove the two statements above, Theorem 7 indeed follows as a corollary of Theorem 1.

We establish the two statements above in turn. The following lemma will be useful.

\section{Lemma 4.}

1. Assume that $\widehat{p} \stackrel{a}{\rightarrow} r$ holds with respect to the operational semantics of CCS for some closed TCCS $S_{M T}$ term $p$ and CCS term $r$. Then $p \stackrel{a}{\rightarrow} p^{\prime}$ holds with respect to the operational semantics of TCCS $S_{M T}$ for some closed TCCS $S_{M T}$ term $p^{\prime}$ such that $\widehat{p^{\prime}}=r$.

2. If $p \stackrel{a}{\rightarrow} p^{\prime}$ holds with respect to the operational semantics of TCC $S_{M T}$ for some closed TCCS $_{M T}$ terms $p, p^{\prime}$ then $\widehat{p} \stackrel{a}{\rightarrow} \widehat{p^{\prime}}$ holds with respect to the operational semantics of CCS.

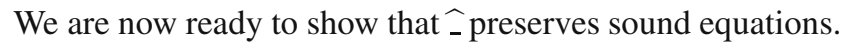

Proposition 1. $t \biguplus_{t} u$ implies $\widehat{t} \biguplus_{b} \widehat{u}$, for all TCCS $S_{M T}$ terms $t, u$.

Proof. It suffices to show that the relation

$$
R=\left\{(\widehat{p}, \widehat{q}) \mid p \biguplus_{t} q, \text { with } p, q \text { closed } \mathrm{TCCS}_{\mathrm{MT}} \text { terms }\right\}
$$

is a strong bisimulation. Indeed, assuming that $R$ is a strong bisimulation, we can show the proposition as follows.

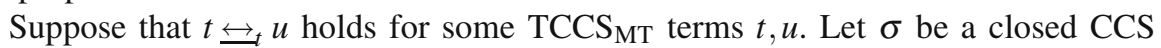
substitution. We shall argue that $\sigma(\widehat{t}) \biguplus_{b} \sigma(\widehat{u})$ holds. This follows because

$-\sigma(\widehat{t})=\widehat{\sigma(t)}$ and $\sigma(\widehat{u})=\widehat{\sigma(u)}$ (by Lemma 1, as $\widehat{-}$ is structural and $\sigma=\widehat{\sigma})$, and $-\widehat{\sigma(t)} \biguplus_{b} \widehat{\sigma(u)}$ (since $\widehat{\sigma(t)} R \widehat{\sigma(u)}$ and $R$ is a strong bisimulation).

So we are left to show that $R$ is indeed a strong bisimulation. This can be easily checked using Lemma 4.

To complete the proof of Theorem 7, we now show that $\widehat{\widehat{Z}}$ is $\mathscr{M}$-reflecting. Since $\widehat{-}$ is the identity function over CCS terms, it suffices to prove the following result. (Note that, since CCS is a reduct of the language $\operatorname{TCCS}_{\mathrm{MT}}$, it makes sense to consider CCS terms modulo $\varliminf_{t}$.)

Proposition 2. The relations $\biguplus_{t}$ and $\biguplus_{b}$ coincide over CCS terms. 
Proof. The relation $\biguplus_{t}$ is included in $\biguplus_{b}$ over the collection of CCS terms by Proposition 1 . The converse inclusion follows because $\leftrightarrows b$ is a timed bisimulation. This can be shown using Lemma 4 and observing that $p \stackrel{\varepsilon(d)}{\rightarrow}$ holds for each closed CCS term $p$ and positive integer $d$.

Since all the provisos of Theorem 1 are met by our reduction, Theorem 7 follows.

\subsection{Other Calculi, Equivalences and Preorders}

There are many other extensions of process algebras in the literature, and each of these languages comes equipped with notions of behavioral equivalence and/or preorder. In this section, we briefly review the results we obtained using our reduction technique for a few such extensions and refer the reader to the extended version of this paper [5] for the full treatment of these cases. Here we limit ourselves to remarking that all the non-finite axiomatizability results covered by the following theorem are proved using $\mathscr{M}$-reflecting reductions to CCS.

Theorem 8. The following process algebras afford no finite (ground-)complete axiomatization: ATP modulo timed bisimilarity [25]; TACS ${ }^{U T}$ modulo the faster-than preorder [16]; TACS ${ }^{L T}$ modulo the MT-preorder [17]; TACS modulo urgent timed bisimilarity [18]; and IMC modulo strong Markovian bisimilarity [15].

\section{Limitations of Our Approach}

As witnessed by the applications described in the previous section, our reduction-based method for proving non-finite axiomatizability results, based on Theorem 1, is widely applicable. Moreover, in all of the applications of Theorem 1 we presented in Section 4, we used CCS modulo bisimilarity as our target language for an $\mathscr{M}$-reflecting reduction. In this section, we give an example of an equational theory within the realm of classic process algebra, whose non-finite axiomatizability cannot be shown in that fashion.

The language $C C S_{\Omega}$ (a variant of the calculus presented in [7]) is obtained by adding the constant $\Omega$ to the fragment of CCS introduced in Section 4. Intuitively, $\Omega$ stands for a process whose behavior is completely unspecified. The operational semantics of $C C S_{\Omega}$ is given by two ingredients: $\stackrel{a}{\rightarrow}$ transitions, which are defined by the same deduction rules used for CCS (thus, $\Omega$ has no outgoing transitions), and a convergence predicate $\downarrow$, which is the least predicate over closed $C C S_{\Omega}$ satisfying the rules given below.

$$
\overline{\mathbf{0} \downarrow} \overline{a \cdot p \downarrow} \frac{p \downarrow q \downarrow}{p+q \downarrow} \frac{p \downarrow q \downarrow}{p \| q \downarrow}
$$

So, for instance $a . \Omega \downarrow$, but neither $\Omega \downarrow$ nor $a \| \Omega \downarrow$ hold. 
The following notion of prebisimilarity is a relevant notion of behavioral preorder in the presence of divergence as adopted in, e.g., [13]. We refer the interested reader to that paper and the references therein for a wealth of results on the semantic theory of $C C S_{\Omega}$ modulo prebisimilarity.

Definition 5. The relation $\check{\varpi}_{\text {pre }}$ is the largest relation over the closed terms of $C C S_{\Omega}$ satisfying the following clauses, whenever $p \check{\sim}_{\text {pre }} q$,

1. for each $p^{\prime}$, if $p \stackrel{a}{\rightarrow} p^{\prime}$ then there exists a $q^{\prime}$ such that $q \stackrel{a}{\rightarrow} q^{\prime}$ and $p^{\prime} \stackrel{\sqsubset}{\sim}$ pre $q^{\prime}$;

2. if $p \downarrow$, then

a. $q \downarrow$ and

b. for each $q^{\prime}$, if $q \stackrel{a}{\rightarrow} q^{\prime}$, then there exists a $p^{\prime}$ such that $p \stackrel{a}{\rightarrow} p^{\prime}$ and $p^{\prime} \stackrel{\sqsubseteq}{\sim}_{p r e} q^{\prime}$.

The relation $\check{\sim}_{\text {pre }}$ is a preorder and a precongruence over closed $C C S_{\Omega}$ terms. Moreover, it coincides with bisimilarity over CCS terms.

Using an argument based on the soundness of the equations in the set $\mathscr{M}$ over $C C S_{\Omega}$ modulo $\check{\sqsubset}_{\text {pre }}$, we can show the following theorem.

Theorem 9. $C C S_{\Omega}$ affords no finite sound and ground-complete axiomatization modulo $\check{\text { pre }}_{\text {. }}$

It is natural to wonder whether the above result can be established, like all those we presented in Section 4, by using CCS modulo bisimilarity as our target language for an $\mathscr{M}$-reflecting reduction. The following theorem shows that this is not possible, and highlights a limitation of our present proof strategy based on reductions to CCS modulo bisimilarity.

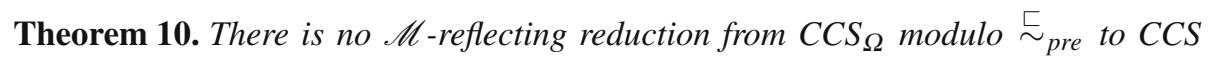
modulo strong bisimilarity.

\section{Conclusions}

In this paper, we have proposed a meta-theorem for proving non-finite axiomatizability results. This theorem can be used to show such results when there exists a reduction from the calculus under consideration to a calculus for which non-finite axiomatizability is known. If the reduction is defined structurally (in the sense of Definition 3), then one only needs to prove that the reduction preserves sound (in)equalities and that it reflects a set of "difficult" (in)equations that form the core of the non-finite axiomatizability result over the target calculus. We have shown seven new non-finite axiomatizability results in process algebra by applying our meta-theorem and reducing different calculi (modulo their respective notion of equivalence or preorder) to a subset of CCS. We intend to apply our reduction technique to obtain several other new non-finite axiomatizability results in process algebra. 
The above-mentioned conditions on the reductions can be established following similar lines for the different calculi and different notions of (pre)congruence studied in this paper. The resulting proofs are substantially more concise and simpler than typical proofs of non-finite axiomatizability. We believe that the proofs of the aforementioned two conditions can be further simplified if one commits to particular models such as those given by Plotkin-style SOS rules. A promising future research direction is to study whether one can apply our meta-theorem in conservative and orthogonal language extensions. Using the SOS meta-theory, one can seek sufficient syntactic conditions on the reduction function that would automatically provide us with the properties required by our meta-theorem. Furthermore, in this paper, we pointed out a limitation of our meta-theorem by presenting a non-finite axiomatizability result that cannot be proved using our general strategy of reducing calculi to CCS. Studying the roots of such limitations may lead to improvements upon the meta-theorem presented in this paper.

Acknowledgements The work of Aceto, Ingolfsdottir and Mousavi has been partially supported by the projects "The Equational Logic of Parallel Processes" (nr. 060013021), "A Unifying Framework for Operational Semantics" (nr. 070030041) and "New Developments in Operational Semantics" (nr. 080039021) of the Icelandic Research Fund.

\section{References}

1. L. Aceto, T. Chen, W. Fokkink, and A. Ingolfsdottir. On the axiomatizability of priority. Proceedings of Automata, Languages and Programming, 33rd International Colloquium, ICALP 2006, Venice, Italy, July 10-14, 2006, Part II, volume 4052 of LNCS, pages 480-491, Springer, 2006.

2. L. Aceto, W. Fokkink and A. Ingolfsdottir. Ready to preorder: Get your BCCSP axiomatization for free! Proceedings of CALCO'07, volume 4624 of LNCS, pages 338-367. Springer, 2007.

3. L. Aceto, W. Fokkink, A. Ingolfsdottir, and B. Luttik. CCS with Hennessy's merge has no finite equational axiomatization. Theoretical Computer Science, 330(3):377-405, 2005.

4. L. Aceto, W. Fokkink, A. Ingolfsdottir, and B. Luttik. Finite equational bases in process algebra: Results and open questions. In A. Middeldorp, V. van Oostrom, F. van Raamsdonk, and R. C. de Vrijer, editors, Processes, Terms and Cycles: Steps on the Road to Infinity, Essays Dedicated to Jan Willem Klop, on the Occasion of His 60th Birthday, volume 3838 of LNCS, pages 338-367. Springer, 2005.

5. L. Aceto, W. Fokkink, A. Ingolfsdottir, and M.R. Mousavi. Lifting non-finite axiomatizability results to extensions of process algebras. Technical Report CSR-08-05, TU/Eindhoven, 2008.

6. L. Aceto, W. Fokkink, A. Ingolfsdottir, and S. Nain. Bisimilarity is not finitely based over BPA with interrupt. Theoretical Computer Science, 366(1-2):60-81, 2006.

7. L. Aceto and M. Hennessy. Termination, deadlock, and divergence. Journal of the ACM, 39(1):147-187, 1992.

8. L. Aceto, A. Ingolfsdottir and M. R. Mousavi. Impossibility results for the equational theory of Timed CCS. Proceedings of CALCO'07, volume 4624 of LNCS, pages 80-95, Springer, 2007.

9. J.A. Bergstra and J.W. Klop. Process algebra for synchronous communication. Information and Control, 60(1-3):109-137, 1984.

10. S.C.C. Blom, W.J. Fokkink, J.F. Groote, I. van Langevelde, B. Lisser, and J.C. van de Pol. mCRL: A toolset for analysing algebraic specifications. Proceedings of CAV'01, volume 2102 of LNCS, pages 250-254, Springer, 2001. 
11. S. L. Bloom and Z. Ésik. Nonfinite axiomatizability of shuffle inequalities. Proceedings of TAPSOFT'95, volume 915 of LNCS, pages 318-333. Springer, 1995.

12. Z. Ésik and M. Bertol. Nonfinite axiomatizability of the equational theory of shuffle. Acta Informatica, 35(6):505-539, 1998.

13. M. Hennessy. A term model for synchronous processes. Information and Control, 51(1):58-75, 1981.

14. M. Hennessy. Algebraic Theory of Processes. Foundations of Computing Series. MIT Press, 1988.

15. H. Hermanns. Interactive Markov Chains and the Quest for Quantified Quality. Volume 2428 of LNCS, Springer, 2002.

16. G. Lüttgen and W. Vogler. Bisimulation on speed: worst-case efficiency. Information and Computation, 191(2): 105-144, 2004.

17. G. Lüttgen and W. Vogler. Bisimulation on speed: Lower time bounds. Proceedings of FOSSACS'04, volume 2987 of LNCS, Springer, 2004.

18. G. Lüttgen and W. Vogler. Bisimulation on speed: A unified approach. Theoretical Computer Science, 360(1-3):209-227, 2006.

19. R. Milner. Communication and Concurrency. Prentice Hall, 1989.

20. F. Moller. Axioms for Concurrency. Ph.D. Thesis, University of Edinburgh, 1989.

21. F. Moller. The nonexistence of finite axiomatisations for CCS congruences. In Proceedings, Fifth Annual IEEE Symposium on Logic in Computer Science, pages 142-153. IEEE Computer Society, 1990.

22. F. Moller. The importance of the left merge operator in process algebras. In Proceedings of ICALP'90, volume 443 of LNCS, pages 752-764. Springer, 1990.

23. F. Moller and C.M.N. Tofts. A temporal calculus of communicating systems. In Proceedings of CONCUR'90, volume 458 of $L N C S$, pages 401-415. Springer, 1990.

24. F. Moller and C. Tofts. Relating processes with respect to speed. In Proceedings of CONCUR '91, volume 527 of $L N C S$, pages 424-438. Springer, 1991.

25. X. Nicollin, J. Sifakis. The algebra of timed processes, ATP: Theory and application. Information and Computation, 114(1):131-178, 1994.

26. M. Sipser. Introduction to the Theory of Computation. 2nd Ed., PWS Publishing, 2005.

27. W. Yi. Real-time behaviour of asynchronous agents. In Proceedings of CONCUR'90, volume 458 of $L N C S$, pages 502-520. Springer, 1990. 